\title{
A Prospective Blinded Study of Endoscopic Ultrasound Elastography in Liver Disease: Towards a Virtual Biopsy
}

\author{
Allison R. Schulman ${ }^{1}$, Ming V. Lin ${ }^{1,2}$, Anna Rutherford ${ }^{1,2}$, Walter W. Chan ${ }^{1,2}$ and Marvin Ryou, ${ }^{1,2}$ \\ ${ }^{1}$ Division of Gastroenterology, Hepatology and Endoscopy, Brigham and Women's Hospital, Boston, MA, ${ }^{2}$ Department of Medicine, Harvard Medical
} School, Boston, MA, USA

Background/Aims: Liver biopsy has traditionally been used for determining the degree of fibrosis, however there are several limitations. Endoscopic ultrasound (EUS) real-time elastography (RTE) is a novel technology that uses image enhancement to display differences in tissue compressibility. We sought to assess whether liver fibrosis index (LFI) can distinguish normal, fatty, and cirrhotic liver tissue.

Methods: A total of 50 patients undergoing EUS were prospectively enrolled. RTE of the liver was performed to synthesize the LFI in each patient. Univariate and multivariable analyses were performed. Chi-square and $t$-tests were performed for categorical and continuous variables, respectively. A $p$-value of $<0.05$ was considered significant.

Results: Abdominal imaging prior to endoscopic evaluation suggested normal tissue, fatty liver, and cirrhosis in 26,16 , and 8 patients, respectively. Patients with cirrhosis had significantly increased mean LFI compared to the fatty liver $(3.2$ vs. $1.7, p<0.001)$ and normal $(3.2$ vs. $0.8, p<0.001)$ groups. The fatty liver group showed significantly increased LFI compared to the normal group $(3.8$ vs. $1.4, p<0.001)$. Multivariable regression analysis suggested that LFI was an independent predictor of group features $(p<0.001)$.

Conclusions: LFI computed from RTE images significantly correlates with abdominal imaging and can distinguish normal, fatty, and cirrhotic-appearing livers; therefore, LFI may play an important role in patients with chronic liver disease.

Clin Endosc 2018;51:181-185

Key Words: Endosonography; Endoscopic ultrasound; Elasticity imaging techniques; Chronic liver disease; Advanced imaging

\section{INTRODUCTION}

Liver biopsy has traditionally been used for determining the index fibrosis in patients with chronic liver disease. However, its usefulness is limited by invasiveness, cost, sampling error, and the difficulty of monitoring the progression of fibrosis over time. $^{1-3}$

Over the past several years, there has been significant inter-

Received: June 9, 2017 Revised: August 29, 2017

Accepted: October 26, 2017

Correspondence: Marvin Ryou

Division of Gastroenterology, Hepatology and Endoscopy, Brigham and Women's Hospital, 75 Francis Street, ASB II, Boston, MA 02115, USA

Tel: +1-617-525-8266, Fax: +1-617-264-6342, E-mail: mryou@bwh.harvard.edu ORCID: https://orcid.org/0000-0001-8120-6497

(c) This is an Open Access article distributed under the terms of the Creative Commons Attribution Non-Commercial License (http://creativecommons.org/ licenses/by-nc/3.0) which permits unrestricted non-commercial use, distribution, and reproduction in any medium, provided the original work is properly cited. est in assessing the utility of non-invasive techniques for the evaluation of liver disease. ${ }^{3,4}$ The most promising and prevailing technique thus far is tissue elastography, a method that can effectively determine liver stiffness or compressibility and has been validated for the staging of liver fibrosis. ${ }^{5,6}$ One form of elastography, called transient elastography (FibroScan ${ }^{\mathrm{TM}}$ ), is a now widely used transabdominal probe-based device; however, it is less sensitive in patients with ascites, thick subcutaneous fat, narrow intercostal spaces, and hepatic atrophy. ${ }^{7-10}$ Furthermore, significant intra- and inter-observer variability has been found based on the degree of probe compression during data collection, along with decreased accuracy in diagnosing moderate fibrosis. $2,4,7,11,12$

Real-time elastography (RTE) is another type of elastography that may be more sensitive than transient elastography in diagnosing the degree of liver fibrosis. This novel technology uses image enhancement to display differences in tissue 
compressibility. Liver fibrosis index (LFI) can be determined using this method, with validation studies demonstrating that an LFI greater than 2.56 correlates with METAVIR scores of F4. ${ }^{13}$ Additionally, RTE does not require ultrasound probe compression for image acquisition, thereby decreasing the variability associated with transient elastography. ${ }^{13-15}$

While studies to date have focuses on transabdominal RTE, endoscopic ultrasound (EUS) RTE may provide a simplified treatment option for patients with liver disease, many of whom are already undergoing endoscopic examination for variceal screening and surveillance. Moreover, EUS RTE may be theoretically more sensitive than transabdominal RTE in the detection of the degree of liver fibrosis because the EUS approach requires signal penetration through a thin gastric wall (usually at most $5 \mathrm{~mm}$ ) compared to the transabdominal approach, which requires signal penetration through the thick abdominal wall (usually several centimeters) (Fig. 1). In this pilot study, we sought to assess whether LFI obtained via EUS RTE could distinguish normal, fatty, and cirrhotic liver tissue in a cohort of patients with chronic liver disease.

\section{MATERIALS AND METHODS}

\section{Study design}

This was a prospective cohort study. The basic design is shown in Fig. 1. Consecutive patients requiring EUS were prospectively enrolled. The endoscopist was blinded to the patients' history of liver disease. The study was approved by the Partners Healthcare Institutional Review Board prior to inception. Fifty consecutive patients who had undergone abdominal imaging performed within six months of EUS RTE that indicated normal, fatty, or cirrhotic liver tissue were then enrolled. One experienced operator performed all RTE examinations. At the time of the endoscopy, the proceduralist was blinded to the severity and etiology of chronic liver disease, and the LFI was calculated following completion of the procedure. All procedures were performed under propofol anesthesia. Demographic information detailed in the medical record was available for all patients.

\section{Study population and assembly of patients}

Patients were recruited from the Gastroenterology and Hepatology clinics at Brigham and Women's Hospital. All patients were referred by an experienced, board-certified gastroenterologist and/or hepatologist and had a clinical indication to undergo EUS. These patients were contacted by phone by a member of the study staff if additional questions arose, and the procedure was discussed in detail at that time. If the patient agreed to undergo the procedure, he/she formally con-

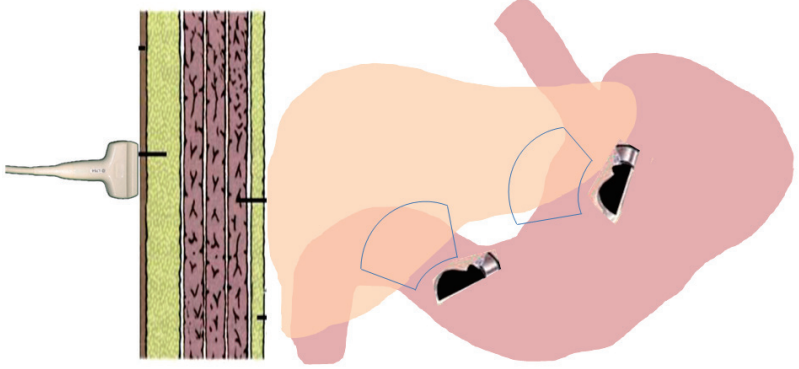

Fig. 1. Schematic demonstration of endoscopic ultrasound-based real time elastography, which can evaluate both the right and left liver through the gastrointestinal wall compared to trans-abdominal elastography, which requires signal transmission through the thick abdominal wall.

sented at the time of the endoscopy. Inclusion and exclusion criteria are shown in Fig. 2.

\section{Data acquisition}

The RTE machine utilized for this study was the Preirus ${ }^{\mathrm{TM}}$ EUS system (Pentax, Montvale, NJ, USA). This modality displays elastic information and the strain of the tissue, which is calculated from the tissue displacement produced by internal compression and relaxation of the heart.

During EUS, proprietary analysis software (Hitachi Medical Systems Europe) was used to calculate six RTE images of the left liver via a transgastric approach. These images were collected for each patient and analyzed with proprietary software to evaluate nine image features (mean of the relative strain value, standard deviation of the relative strain value, ratio of blue area in the analyzed region, complexity of the blue area, kurtosis of the strain histogram, skewness of the strain histogram, entropy, inverse difference moment, and angular second moment) and to synthesize a single value known as the LFI.

\section{Outcomes}

We sought to determine whether LFI obtained via EUS RTE could distinguish normal, fatty, and cirrhotic liver tissue in accordance with previously obtained abdominal imaging.

\section{Statistical analysis}

A univariate analysis was performed to compare baseline characteristics between patients with normal, fatty, and cirrhotic liver imaging. Chi-square analyses were performed for all binary and categorical variables, and $t$-tests were performed for continuous variables. Pairwise comparisons between groups were performed using $t$-tests and ANOVA, and reported as mean \pm standard error (SE), with a two-sided $p$-value of $<0.05$ considered significant. Multivariable regression analysis was performed to determine whether LFI was associated with cirrhosis as determined based on abdominal 


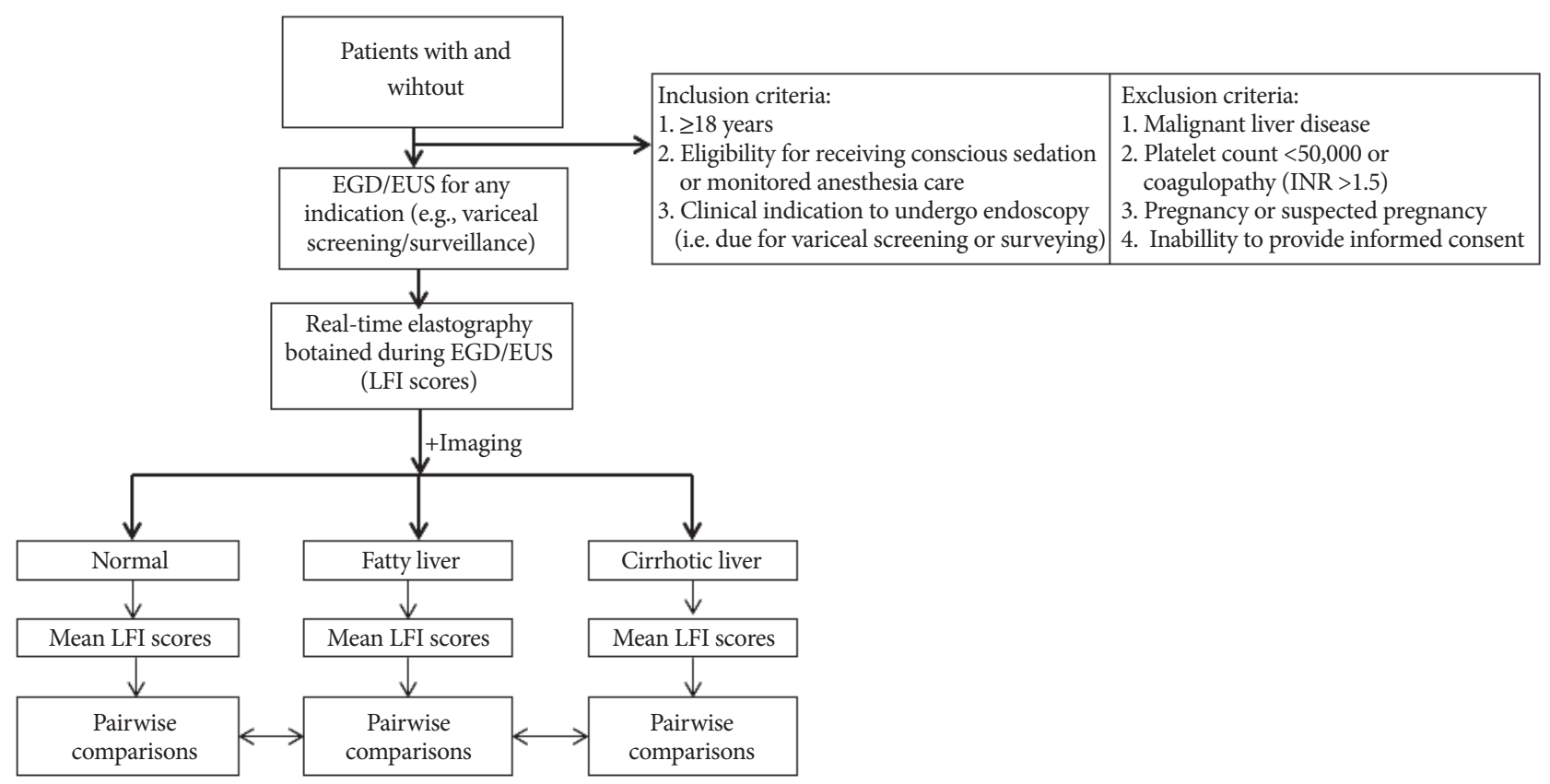

Fig. 2. Flowchart showing the basic study design. EGD, esophagogastroduodenoscopy; EUS, endoscopic ultrasound; LFI, liver fibrosis index; INR, international normalized ratio.
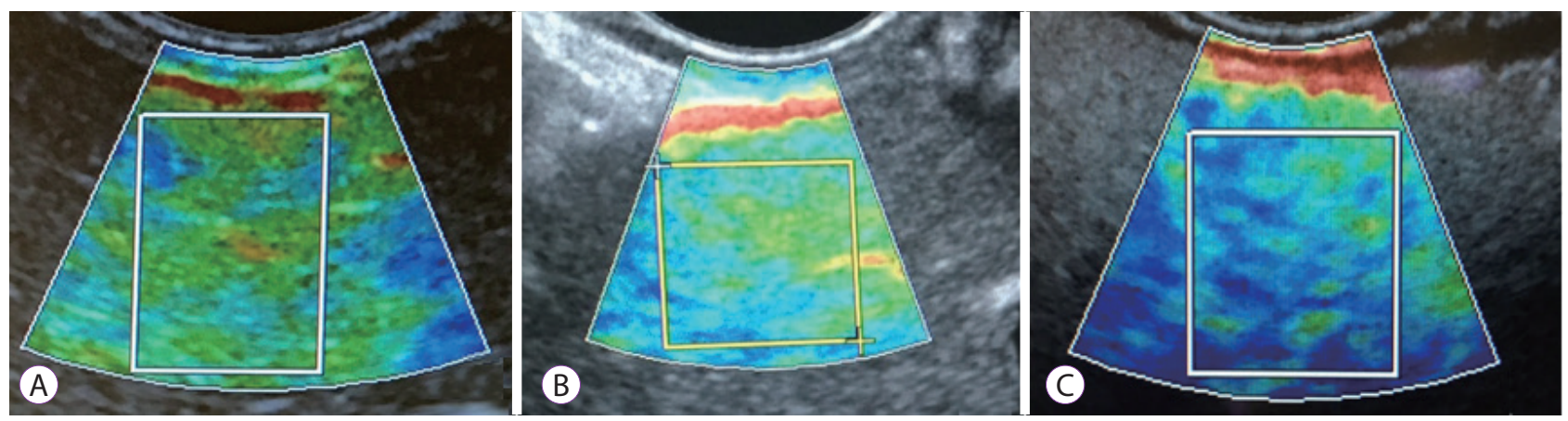

Fig. 3. Representative endoscopic ultrasound real-time elastography images in patients with normal $(A)$, fatty $(B)$, and cirrhotic (C) liver tissue on cross-sectional imaging.

imaging findings. All statistical analyses were performed using the SAS 9.4 statistical package (SAS Institute Inc., Cary, NC, USA).

\section{RESULTS}

A total of 50 consecutive patients with clinical indications to undergo EUS were enrolled (Fig. 2). All patients had abdominal imaging prior to endoscopic evaluation, and prior biopsy results indicated normal liver, fatty liver, and cirrhosis in 26,16 , and 8 patients, respectively. Baseline characteristics for each study group and the etiologies of cirrhosis are shown in Table 1.

All patients successfully completed the procedure. There were no intra- or peri-procedural adverse events. Mean pro- cedure time was 34 minutes (+/- $5.4 \mathrm{SE})$. The RTE component accounted for a mean 5 minutes (+/- 1.7 SE) of the total procedure time. Representative images from each of the three groups are shown in Fig. 3. The cirrhotic group showed significantly increased mean LFI scores compared to the fatty liver (3.2 vs. $1.7, p<0.001$ ) and normal (3.2 vs. $0.8, p<0.001$ ) groups (Fig. 4). The fatty liver group had significantly increased LFI scores compared to the normal group (3.8 vs. 1.4, $p<0.001$ ) (Fig. 4).

Multivariable regression suggested that LFI was an independent predictor of group features $(p<0.001)$ when adjusting for other variables including age and sex. Using the previously published LFI cut-off of 2.56, the area under the receiver operator characteristic (ROC) curve for LFI in predicting cirrhosis on imaging was 0.865 , which suggests strong predictive value (Fig. 5). 
Table 1. Baseline Characteristics of Patients Included in the Study

\begin{tabular}{|c|c|c|c|}
\hline Variable & $\begin{array}{c}\text { Normal } \\
(n=26) \\
n(\%)\end{array}$ & $\begin{array}{c}\text { Fatty } \\
(n=16) \\
n(\%)\end{array}$ & $\begin{array}{c}\text { Cirrhosis }^{\mathrm{a})} \\
(n=8) \\
n(\%)\end{array}$ \\
\hline Age $($ mean $\pm S D)$ & $59.3 \pm 18$ & $58.3 \pm 17$ & $64.2 \pm 14$ \\
\hline Sex, male & $12(43)$ & $7(44)$ & $4(50)$ \\
\hline $\begin{array}{l}\mathrm{BMI} \mathrm{kg} / \mathrm{m}^{2} \\
\quad(\text { mean } \pm \mathrm{SD})\end{array}$ & $29.0 \pm 6$ & $30.8 \pm 7$ & $28.3 \pm 4$ \\
\hline Hypertension & $10(37)$ & $12(71)$ & $7(88)$ \\
\hline Hyperlipidemia & $4(15)$ & $9(53)$ & $4(50)$ \\
\hline Diabetes & $1(4)$ & $4(24)$ & $6(75)$ \\
\hline Tobacco use & $4(15)$ & $3(18)$ & $4(50)$ \\
\hline Alcohol use & $9(33)$ & $5(29)$ & $5(63)$ \\
\hline
\end{tabular}

SD, standard deviation; BMI, body mass index.

${ }^{\text {a) }}$ Etiology of cirrhosis: alcoholic $(n=2)$; alcoholic+hepatitis C $(n=2)$; alcoholic+hepatitis B $(n=1)$; hepatitis $\mathrm{B}(n=1)$; non-alcoholic steatohepatitis $(n=2)$.

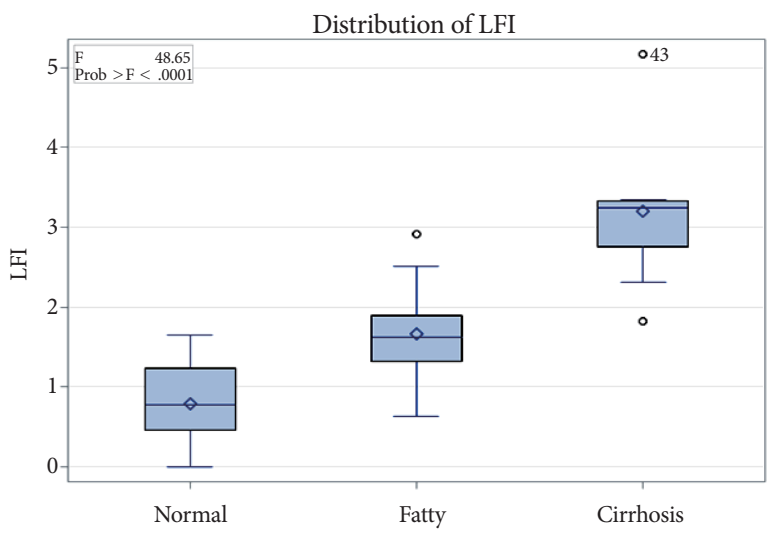

Fig. 4. Comparison of liver fibrosis index (LFI) scores between the normal, fatty, and cirrhotic liver imaging (ANOVA).

\section{DISCUSSION}

Cirrhosis represents a late stage of progressive hepatic fibrosis, in which healthy tissue is replaced by scar tissue, leading to hepatic dysfunction. Liver biopsy has traditionally been used for determining the index of liver fibrosis using a METAVIR score ranging from F0 (no fibrosis) to F4 (cirrhosis). However, the usefulness of liver biopsy is limited by invasiveness, cost, potential complications, and difficulty of repeated procedures for the monitoring of changes over time. Furthermore, a standard liver biopsy sample lacks accuracy due to sampling error, $^{3}$ and its histologic interpretation is prone to substantial subjectivity, leading to discordance in staging in up to $40 \%$ of patients. ${ }^{1-4}$

RTE is a novel technology that offers an alternative method

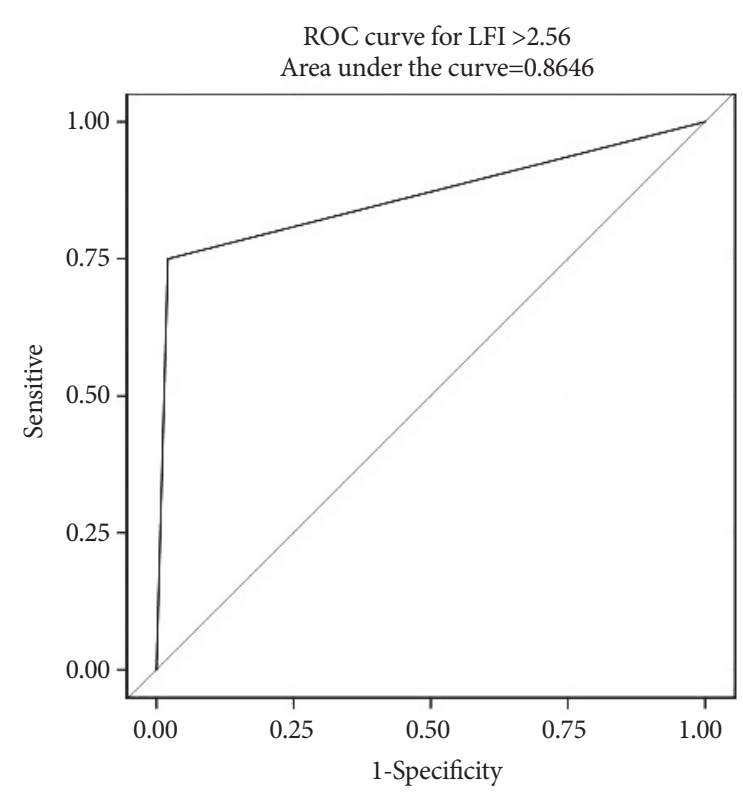

Fig. 5. Area under the receiver operator characteristic (ROC) curve for the prediction of cirrhosis on imaging by liver fibrosis index (LFI).

of image enhancement to display the differences in compressibility between tissues. As the name implies, the images are obtained in real time, allowing for the visualization of a two-dimensional strain image induced by internal heartbeats. The pattern of the induced strain image becomes patchy as fibrosis progresses. Previous work using transabdominal RTE has confirmed that LFI computed from RTE images highly correlates with the METAVIR stages of hepatic fibrosis, with significant differences existing between all stages. This relationship remains valid even in the presence of inflammation.

In this clinical pilot study, we assessed whether LFI obtained via EUS RTE could distinguish normal, fatty, and cirrhotic liver tissue in a cohort of patients undergoing EUS. We found that LFI computed from RTE images significantly correlated with abdominal imaging, and the ANOVA confirmed statistically significant differences between the normal, fatty, and cirrhotic-appearing livers. Furthermore, our multivariable regression analysis suggested that LFI was an independent predictor of group features when adjusting for other variables. Using the previously published LFI cut-off of 2.56 , the area under the ROC curve for predicting cirrhosis on imaging with LFI demonstrated a very strong predictive value.

RTE has several advantages over transient elastography. First, since it does not have the same limitations as transient elastography, it can be used in all patients. It has also been demonstrated to be more sensitive than transient elastography in diagnosing the degree of liver fibrosis, with LFI $>2.56$ correlating with METAVIR scores of F4. ${ }^{14}$ Additionally, RTE does not require ultrasound probe compression for image 
acquisition, which attenuates the variability associated with transient elastography. Additionally, unlike transient elastography, which can only be performed transabdominally, RTE can be performed transabdominally or endoscopically (i.e., via an echo ultrasound probe) and can therefore be employed at the time of screening and surveying for esophageal varices in patients with chronic liver disease. Finally, EUS RTE may be potentially advantageous in patients for whom transabdominal imaging would be inaccurate, i.e., those with ascites or thick subcutaneous fat.

There are several limitations to our study. First, the small number of patients with cirrhosis may limit the power to detect differences and to achieve more extensive controlling of variables with statistical significance. Second, we did not have biopsy data on all patients; therefore, the patients' livers were labeled a priori as normal, fatty, and cirrhotic based on imaging studies. Third, all of our procedures were performed by a single proceduralist with expertise in therapeutic endoscopy. While this helped to standardize the results obtained from the RTE, it is unclear if this association would be as significant or consistent in users with less experience in therapeutic endoscopy.

The results of the present study are encouraging in that they demonstrate the ability of RTE to discriminate normal, fatty, and cirrhotic liver tissue based on cross-sectional imaging, with LFI showing significant predictive value for cirrhosis. Given these results, further studies are underway to determine the comparison with transabdominal RTE and concomitant liver biopsy, as well as the prediction scores for decompensation.

\section{Conflicts of Interest}

Marvin Ryou is a consultant to Covidien, Inc. and founder of GI Windows, Inc. Other authors have no financial conflicts of interest.

\section{Financial Disclosures}

Marvin Ryou has received consulting fees from Covidien, Inc.

\section{Author Contributions}

Conceptualization: Allison R. Schulman, Ming V. Lin, Anna Rutherford, Walter W. Chan, Marvin Ryou

Data curation: ARS, WWC, MR

Formal analysis: ARS, WWC, MR

Investigation: ARS, MVL, AR, WWC, MR

Methodology: ARS, MVL, AR, WWC, MR
Project administration: ARS, MR

Resources: ARS, WWC, MR

Supervision: MR

Validation: ARS, WWC, MR

Visualization: ARS, WWC, MR

Writing-original draft: ARS, MR

Writing-review\&editing: ARS, MVL, AR, WWC, MR

\section{REFERENCES}

1. Bedossa P, Poynard T. An algorithm for the grading of activity in chronic hepatitis C. The METAVIR cooperative study group. Hepatology 1996;24:289-293.

2. Pang JX, Zimmer S, Niu S, et al. Liver stiffness by transient elastography predicts liver-related complications and mortality in patients with chronic liver disease. PLoS One 2014;9:e95776.

3. Bedossa $P$, Dargère D, Paradis V. Sampling variability of liver fibrosis in chronic hepatitis C. Hepatology 2003;38:1449-1457.

4. Castera L, Forns X, Alberti A. Non-invasive evaluation of liver fibrosis using transient elastography. J Hepatol 2008;48:835-847.

5. Berzigotti A, Ashkenazi E, Reverter E, Abraldes JG, Bosch J. Non-invasive diagnostic and prognostic evaluation of liver cirrhosis and portal hypertension. Dis Markers 2011;31:129-138.

6. Augustin S, Millán L, González A, et al. Detection of early portal hypertension with routine data and liver stiffness in patients with asymptomatic liver disease: a prospective study. J Hepatol 2014;60:561-569.

7. Fraquelli M, Rigamonti C, Casazza G, et al. Reproducibility of transient elastography in the evaluation of liver fibrosis in patients with chronic liver disease. Gut 2007;56:968-973.

8. Castéra L, Foucher J, Bernard PH, et al. Pitfalls of liver stiffness measurement: a 5-year prospective study of 13,369 examinations. Hepatology 2010;51:828-835.

9. Wong GL, Wong VW, Chim AM, et al. Factors associated with unreliable liver stiffness measurement and its failure with transient elastography in the Chinese population. J Gastroenterol Hepatol 2011;26:300-305.

10. Chang PE, Goh GB, Ngu JH, Tan HK, Tan CK. Clinical applications, limitations and future role of transient elastography in the management of liver disease. World J Gastrointest Pharmacol Ther 2016;7:91-106.

11. Kettaneh A, Marcellin P, Douvin C, et al. Features associated with success rate and performance of fibroscan measurements for the diagnosis of cirrhosis in HCV patients: a prospective study of 935 patients. J Hepatol 2007;46:628-634.

12. Lucidarme D, Foucher J, Le Bail B, et al. Factors of accuracy of transient elastography (fibroscan) for the diagnosis of liver fibrosis in chronic hepatitis C. Hepatology 2009;49:1083-1089.

13. Tatsumi C, Kudo M, Ueshima K, et al. Non-invasive evaluation of hepatic fibrosis for type C chronic hepatitis. Intervirology 2010;53:76-81.

14. Robic MA, Procopet B, Métivier S, et al. Liver stiffness accurately predicts portal hypertension related complications in patients with chronic liver disease: a prospective study. J Hepatol 2011;55:1017-1024.

15. Tatsumi C, Kudo M, Ueshima K, et al. Noninvasive evaluation of hepatic fibrosis using serum fibrotic markers, transient elastography (fibroscan) and real-time tissue elastography. Intervirology 2008;51(Suppl 1):27-33. 